\title{
Third party interpretation of raw genetic data: an ethical exploration
}

\author{
Lauren Badalato $^{1,2}$, Louiza Kalokairinou ${ }^{2}$ and Pascal Borry ${ }^{\star, 2}$
}

In the wake of recent regulations targeting direct-to-consumer genetic testing (DTC-GT), an increasing number of websites have emerged that offer consumers alternative means to derive health information from their DTC-GT raw data. While the ethical concerns associated with DTC-GT have been extensively discussed in the literature, the implications of third party interpretation (TPI) websites have remained largely unexplored. Here we sought to describe these services and elucidate their ethical implications in the context of the current DTC-GT debate. We reviewed five popular TPI websites that use SNP-based genomic data to report health-related information: Promethease, Interpretome, LiveWello, Codegen.eu, and Enlis Personal. We found that many of the ethical concerns previously described in DTC-GT also applied to TPI websites, including inadequate informed consent, questionable clinical validity and utility, and lack of medical supervision. However, some concerns about data usage and privacy reported in DTC-GT were less prominent in the five TPI websites we studied: none of them sold or shared user data, and $3 / 5$ sites did not retain data in the long term. In addition, while exaggerated claims and inaccurate advertising have been frequently problematic in DTC-GT, advertising was minimal in the TPI sites we assessed, and 4/5 made no claims of health benefits. Overall, TPI adds a new dimension to the ethical debate surrounding DTC-GT, and awareness of these services will become increasingly important as personal genomics continues to expand. This study constitutes the first detailed ethical analysis of these services, and presents a starting point for further research and ethical reflection.

European Journal of Human Genetics (2017) 25, 1189-1194; doi:10.1038/ejhg.2017.126; published online 23 August 2017

\section{INTRODUCTION}

Direct-to-consumer genetic testing (DTC-GT) refers to genetic tests that are either sold or advertised directly to the public, outside of the traditional healthcare setting. ${ }^{1,2}$ The reporting of health-related genetic information from DTC-GT commercial companies has sparked heated ethical and regulatory debates, which have largely concerned the clinical validity and utility of testing, consumer privacy, claims and advertising and inadequate medical supervision. ${ }^{3}$ These concerns have led numerous professional organizations to establish guidelines outlining their positions on appropriate use and regulation of DTC$\mathrm{GT},{ }^{2,4-6}$ and some countries have adopted legislation that restricts DTC-GT companies from reporting health-related information.,

In the wake of stricter regulation of DTC-GT companies, an increasing number of online services have emerged that provide an alternative means of interpreting personal genetic data. Users can input their genetic data files into these third party interpretation (TPI) websites, which use bioinformatic analysis to generate a report particular to that individual's genomic variants. Most of the websites we observed use single nucleotide polymorphism (SNP)-based raw data derived from DTC-GT companies such as 23 and Me, Ancestry. com, and FamilyTreeDNA, ${ }^{9-11}$ although some allow for processing of whole genome sequencing (WGS) or whole exome sequencing (WES) data, ${ }^{12-14}$ which can also be obtained direct-to-consumer at a greater cost. Some TPI sites provide specific testing related to nutrigenomics as well as methylation and 'detox,' and often suggest supplements to go along with their interpretations (geneticgenie.org, nutrahacker.org). ${ }^{15,16}$
Others primarily assess no-medical traits including ancestry and athletic performance (GEDmatch.com, athletigen.com). ${ }^{17,18}$ There are some sites with a research focus (DIYgenomics, OpenSNP, dna. land), ${ }^{19-21}$ while others provide health data based on genome-wide untargeted analysis. ${ }^{13,14,22-24}$ In this study, we selected five websites that analyse SNP-based genomic data to provide health-related information: Codegen.eu, Interpretome, LiveWello, Enlis Personal and Promethease. Several of these websites characterize themselves as primarily having a literature search function. ${ }^{22,13}$ They operate by querying different publicly available databases such as SNPedia, dbSNP, Genecards, GET-Evidence and matching consumer 'reference SNP cluster IDs' (rsIDs) - the unique SNP identifiers within DTC-GT raw data - with reported disease associations in the medical literature. While the public is free to search these databases independently, these sites make this information much more accessible to the average user. The returned results typically included risk estimates for common complex diseases, sometimes with interpretations that particular SNPs are 'good,' 'bad,' 'pathogenic' or 'risk factors;' reports can also include more serious single-gene disorders or carrier status.

Overall, there is a general paucity of information in the academic literature about TPI services and they have received little attention from regulatory bodies and professional societies. In this article, we seek to describe these services, and review their ethical implications using the on-going ethical debate surrounding DTC-GT as a framework for our discussion. In the following sections we will address issues related to clinical validity and utility; informed consent; medical

\footnotetext{
${ }^{1}$ Department of Genetics, Children's Hospital of Eastern Ontario, Ottawa, Ontario, Canada; ${ }^{2}$ Centre for Biomedical Ethics and Law, Department of Public Health and Primary Care, University of Leuven, Leuven, Belgium

*Correspondence: Dr P Borry, Centre for Biomedical Ethics and Law, Kapucijnenvoer 35 BOX 7001 , Leuven 3000 KU Leuven, Belgium. Tel: +32 16 379517 or +32 16 336946 ; Fax: +32 163369 52; E-mail: Pascal.Borry@med.kuleuven.be

Received 13 February 2017; revised 10 June 2017; accepted 4 July 2017; published online 23 August 2017
} 
supervision; claims, advertising and disclaimers; and privacy/ data usage.

\section{METHODS}

We found the TPI websites through Google searches including terms such as 'interpretation of personal genomic raw data' and 'interpretation of 23 and Me raw data.' We reviewed various blogs, forums and articles and compiled a list of 29 websites that offered to interpret consumer genomic data. Four of these sites were no longer functioning at the time of analysis. We excluded websites that had a strong focus on open sourced research, non-medical traits or genealogy. We also excluded mobile apps, sites that were not consumer-oriented or that primarily analyzed WES/WGS data. The five analyzed TPI sites (Codegen.eu, Interpretome, LiveWello, Enlis Personal, and Promethease) were selected based on the following criteria: they were recurrently mentioned in personal genomics articles, blogs and forums, and they primarily provided health information using SNP-based genomic data. For our assessment, we reviewed the content of these websites, including any sample reports, blogs or published literature they provided.

\section{RESULTS}

\section{Clinical validity and utility}

Questions surrounding the clinical value of results derived from untargeted SNP-based technologies have been central within the DTC-GT ethical debate. ${ }^{4,25,26}$ The TPI websites we assessed also report SNP-based risk estimates, and are hence plagued with many of the same scientific shortcomings. However, an important difference was noted among the TPI sites: rather than combining risk estimates to provide a single risk estimate as many DTC-GT companies have done, ${ }^{26}$ the TPI websites typically presented risks associated with individual SNPs in isolation. Consequently, they avoid some of the scientific concerns associated with the use of unvalidated algorithms to consolidate risk data. ${ }^{26,27}$ This lack of aggregation is not without its drawbacks: reports tend to be extensive and contain long lists of SNPs that can be associated with both increased and decreased risks for the same condition, which could be confusing for users and are unlikely to prove clinically useful. ${ }^{27}$ And yet, receiving such complex, conflicting results may give users a more realistic perspective of the uncertain nature of multifactorial disease prediction. ${ }^{27}$

Numerous regulatory bodies and professional societies have voiced concerns about the issues of clinical validity and utility in DTC-GT, 2,4,5,28 and this became an important factor in recent regulatory actions, including the US Food and Drug administration's actions against several companies in $2013 .{ }^{26}$ Some technology articles and blogs have portrayed TPI websites as a way around such regulations, ${ }^{27,29}$ although none of the TPI websites included in our study openly endorse this. Several do, however, either state or imply that they provide even more health data than traditional DTC-GT. Within their blog, Enlis proclaims that they offer more health related information from 23andMe's raw data than the company was able to offer before the US Food and Drug administration's ban. ${ }^{30}$ They conclude that 'if you want to get the most out of your 23 and Me raw data, you need to get a third-party interpretation. ${ }^{30}$ LiveWello makes a similar, but much more vague statement, 'that people should really have all the information about ALL the genes in their raw data not just some of them. ${ }^{124}$

While the use of SNPs in risk prediction for common complex disease may be scientifically questionable, some reported SNPs represent established pathogenic variants and can thus have more direct clinical relevance. ${ }^{26}$ Consequently, clinically actionable findings may appear in TPI reports; indeed, SNP libraries such as SNPedia are actively working to identify more highly penetrant disease associated variants, ${ }^{31}$ and Enlis Personal notes that it has been working to discover potentially highly pathogenic indel sequences through 'reverse engineering' data from 23 and $\mathrm{Me}^{30}$ While improved clinical validity and utility associated with certain SNPs can be considered a positive evolution in some regards, it raises further concerns related to users obtaining significant medical information without the counselling and support of a healthcare professional, which we will address in a later section.
Essentially, many have doubted the clinical value of the SNP-based predictions for complex disease provided by DTC-GT tests, ${ }^{32}$ and similar scepticism applies to risk predictions from TPI websites. While these technologies yields promise for future research, application to individuals for health purposes is largely considered by experts to be premature $e^{26,33}$ and the current limitations of risk predictions are often not made clear to consumers. ${ }^{34}$ Curiosity about one's genome is not inherently a negative thing, but the provision of potentially meaningless information is a concern, when its value and implications can easily be misperceived. Likewise, providing medically significant and, potentially life-altering information without sufficient preparation or support is also ethically problematic.

\section{Autonomy and informed consent}

Issues related to autonomy and informed consent have also pervaded the ethical debate surrounding DTC-GT, $2,4,35,36$ and many of these concerns similarly apply to TPI. The informed consent process was minimal in all of the TPI websites we assessed: none of the sites had a formal consent form, although several sites had check boxes that had to be agreed before submitting data (Table 1). The potential nature of the information obtained (carrier status, disease risk estimates, pathogenic variants) or the personal impact it may have on users, is not clearly expressed on any of the third party websites.

When we contemplate what constitutes appropriate informed consent, it is also important to consider the prior genetic literacy of the users of TPI websites, and there is little data available on this currently. One may postulate that those using such TPI websites likely have a greater understanding of genomics than the average DTC-GT consumer, given their propensity to seek out these resources. However, it is also possible that the characteristics of early adopters might change as TPI services become increasingly popular. Further study is needed to determine the characteristics of this user population to better understand the level of knowledge and understanding of individuals interested in these services.

Finally, the lack of accountability regarding data ownership and consent is also concerning in both DTC-GT ${ }^{2,4,33,37}$ and in TPI. As opposed to clinical laboratories where samples are confirmed to belong to the appropriate patient, no such safeguards are in place for saliva samples being submitted at home, or raw data submitted online. Thus, it is nearly impossible to prevent the inappropriate submission from an individual who did not, or could not, give consent. Among the TPI websites we reviewed, only one, LiveWello, contained a statement that its users needed to be 18 years of age or older to use its services; ${ }^{38}$ the other sites posted no age restrictions. LiveWello also contains a statement that 'you may provide only information that you own or have the right to use, ${ }^{138}$ The other four websites make no mention about the submission of another party's data. Thus, there are similar concerns in TPI regarding the potential to analyze another's data without proper consent, and there were no discernible safeguards to prevent this from occurring.

Overall, while there may be potential for some DTC-GT and TPI to enhance consumer autonomy, the freedom to choose this option becomes meaningless if users are not adequately informed of the risks, benefits and limitations of such testing. ${ }^{4,34}$ Insufficient, incomprehensible or misleading information represents a failure of informed consent, and undermines autonomy. ${ }^{39}$ The process of informed consent in obtaining health related information from the third party websites was minimal to absent, and raises concerns that users may be participating without truly understanding the risks, benefits and limitations of having their raw data analysed.

\section{Medical supervision}

The problem of inadequate informed consent is further intensified by the lack of medical supervision in DTC-GT and TPI. Concerns about potential misinterpretation of results, inappropriate or absent follow-up care and the potential impact on public health care systems have also been raised. ${ }^{2}$ These issues are similarly pertinent in the case of TPI services. Of the TPI websites we analysed, four of five made some mention of discussing results or any medical issues with a healthcare provider, usually in the context of making healthrelated decisions. ${ }^{13,23,24,40}$ The practice of providing health information but 


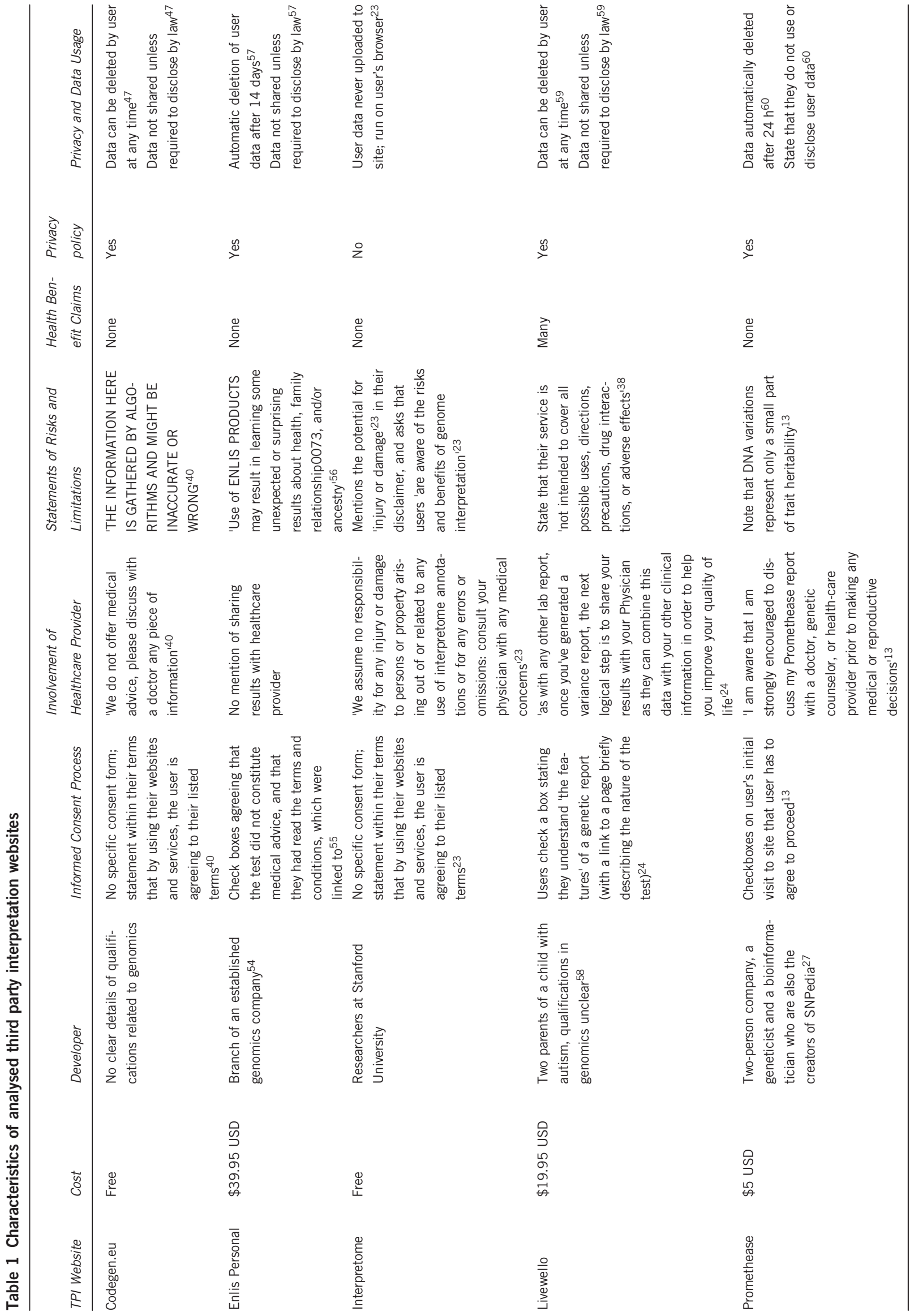


deferring discussion of results to the user's healthcare provider is problematic for several reasons. Most family physicians are ill equipped to deal with genetic information, and may also misinterpret it or misperceive its relevance. ${ }^{41}$ While genetic literacy in primary care should be encouraged overall, ${ }^{4}$ it is also important for any providers of genetic interpretations to supply physicians with sufficient and unbiased information to understand the results. ${ }^{2,42}$ Also, because of the questionable clinical validity and utility of many non-clinical genetic test results, physicians have often been advised not to change clinical management based on these tests. ${ }^{25,43}$ It is concerning that, similar to many DTC-GT companies, the reviewed TPI websites unanimously state that their testing is not for medical purposes, but some paradoxically recommend discussing the results with one's healthcare provider (Table 1). The Canadian College of Medical Genetics (CCMG) condemned this practice in DTC-GT, proclaiming that denying that tests are for health purposes, then suggesting that consumers review them with their physicians was 'insufficient and inappropriate. ${ }^{2}$ Finally, several professional bodies ${ }^{2,4}$ have raised concerns that individuals seeking explanations or follow-up for their DTC-GT will present a cost to public healthcare systems through unnecessary investigations and follow-up. ${ }^{2}$ By presenting users with health related results, TPI services may pose a similar risk.

Overall, the risks associated with lack of medical supervision are common issues among many DTC-GT companies and the TPI services we studied. Insufficient informed consent, lack of counselling, inappropriate follow-up, and justice in public healthcare systems are among the key concerns identified.

\section{Claims, advertising, and disclaimers}

Aside from inadequate informed consent, there have been significant concerns about exaggerated and misleading advertising on the part of DTC-GT companies, ${ }^{5}$ and that there is a general imbalance of the advertised benefits versus stated risks. ${ }^{42,44}$ Compared with the DTC-GT companies reviewed by Singleton et al., ${ }^{44}$ the TPI websites we reviewed made relatively few claims. Promethease $^{13}$ and Interpretome $e^{23}$ are fairly minimalist in their construction, with a simple text-based design and no promotional material. Enlis Personal and Codegen.eu describe the exploratory use of their services and the generation of health information, but do not make claims of health benefits. $^{22,14}$

LiveWello, on the other hand, does make several claims of benefit; for those with chronic diseases, it claims that 'Getting to the root cause, will help facilitate recovery by tailoring any treatment plans and lifestyle changes your Doctor recommends specifically to your body. ${ }^{24}$ They also claim that 'Research is implicating certain genetic mutations in a woman's ability to conceive and sustain a viable pregnancy. The good news is that there are treatments available... ${ }^{24}$ but give no reference to what data they are referring to in this statement. Lastly, they make a similarly nebulous statement in a section labelled 'Preparing for the arrival of your new baby,' that 'With Genetic information, awareness of any potential pitfalls that your child could encounter will help parents make better healthcare and lifestyle choices for their children. ${ }^{24}$ It is unclear from these statements how this type of testing would (or could) be used to 'help' in these situations. Such claims could promote a false hope or unrealistic expectations, similar to previously described concerns about advertising in DTC-GT. ${ }^{44}$

While TPI websites overall had fewer exaggerated claims than many DTCGT companies, there was also a paucity of risk information presented: potential hazards associated with receiving genetic information, including anxiety, genetic discrimination, or impact on family members, ${ }^{5}$ were rarely described on the TPI websites we analysed, and their statements of the risks and limitations were often vague and ambiguous (Table 1). However, some limitations of testing were mentioned to some extent on all of the websites (Table 1).

Overall, relative to reports on DTC-GT websites, ${ }^{44}$ the TPI sites we reviewed tended to have fewer exaggerated advertising claims, less promotional material, and less skewed information. Instead, they tend toward having a general paucity of information: along with fewer claims of benefit, there were minimal mentions of risk and vague disclaimers stating limitations. Thus, while fraudulent or misleading advertising was not a significant issue in most of the TPI websites we studied, insufficiency of information remains a concern.

\section{Privacy and data usage}

Privacy, protection of consumer data, and data usage have been key issues within the ethical debate surrounding DTC-GT. Health information derived by these companies is not subject to the same privacy standards as medical records, ${ }^{5}$ and there is an absence of uniform standards and frequent lack of transparency among DTC-GT privacy practices. ${ }^{45,46}$ Of the TPI websites we analysed, four had a specific privacy policy, and the remaining one $\left(\right.$ Interpretome ${ }^{23}$ ) described their means for protecting privacy within their terms and conditions (Table 1). Risks associated with breach of privacy were not mentioned on any of the websites. However, three out of five TPI services did not store data for the long term (Table 1), including one that did not require data upload at all. Some form of statement regarding data protection was included on all four sites that required data upload, although some of these were rather vague and noted possibilities of privacy breaches.

LiveWello and Codegen.eu provided platforms to facilitate sharing personal results with one's physician, ${ }^{24,47}$ but did not encourage public data sharing (Table 1). However, it is important to note that public data sharing is among the primary functions of some other research oriented services that provide TPI but were not included in our analysis, ${ }^{20,21,48}$ and previously discussed issues of privacy, re-identification, and implications for family members ${ }^{49}$ may be more relevant in these scenarios.

Overall, privacy concerns among the TPI services we analysed included vague, non-specific privacy policies, some risk of breach of privacy, and a lack of discussion of the risks associated with privacy breach. However, there were some protective features observed: several TPI services did not retain user data, which decreases the downstream possibility of re-identification or change of data ownership. Similarly, there was no indication that any of the five TPI services we studied sold, shared or disclosed user data (Table 1).

\section{CONCLUSIONS}

Here we have reviewed many of the ethical issues that have been proposed in DTC-GT, and discussed how they relate to the more recent development of TPI of raw data generated by DTC-GT companies. We observed many similarities in the ethical concerns surrounding DTC-GT and TPI, including inadequate informed consent, questionable clinical validity and utility, and lack of medical supervision. A number of differences were also noted: namely, most of the TPI services we studied contained few advertisements and claims, none shared or sold user data, and most did not retain data in the long-term.

There was also significant variation among the TPI services we analysed as highlighted above: some of the websites, including LiveWello $^{50}$ and Enlis Personal, ${ }^{14}$ had a more commercial appearance similar to DTC-GT companies. Their methods were less transparent, and LiveWello made substantial health claims. ${ }^{24}$ Promethease and Interpretome, ${ }^{23,13}$ on the other hand, were minimalist websites, made no claims, and were transparent in their mining of open-sourced data, each having a publication detailing their methods. ${ }^{31,51}$ Some were free, while others were associated with a cost. Thus, while we observed some common tendencies among the TPI websites we studied, these other aspects were more difficult to generalize.

Similarly, we wish to highlight that the five TPI services we analysed represent a small subset of the available resources, and that there are a vast number of services providing TPI of SNP data for an array of purposes. Like DTC-GT, ${ }^{34}$ the ethical issues associated with different TPI will inevitably vary depending on the purpose of testing and method of analysis. Thus, our study is limited in that we do not discuss all forms of TPI, but by assessing of some of the more common websites in detail, we provide a useful starting point for ethical discussion of these services. Further studies are needed to elucidate the full spectrum of available TPI, and how the aforementioned ethical implications vary among them. 
While many of the TPI websites describe themselves more in terms of literature retrieval and genome exploration, the fact remains that their reports contain medical information, and users are likely to perceive it as such. Reducing personal health information to data points is ethically problematic, and the impacts these results could have on and individual's conception of health, personal identity and family structures remain important considerations.

As the cost of genetic technologies have decreased, so too has the barrier to market entry into the DTC-GT. ${ }^{5}$ For TPI services, the barrier is almost non-existent: one only needs the necessary bioinformatics knowledge and a computer to design such a service. There is no specialized laboratory equipment required, the required data is open access, and sites can be created from anywhere in the world. Given these characteristics, any regulation of TPI will likely be difficult, and its existence complicates the attempts of regulatory bodies to limit provision of health information DTC. ${ }^{52}$

Similarly, as personal genomics expands, it is likely that such services will become increasingly common; as whole genome and whole exome sequencing become more available DTC, TPI will continue to follow suit. ${ }^{53}$ This will likely intensify the ethical debate, as these technologies carry an increased likelihood of identifying high penetrance pathogenic variants, or variants of unknown significance (VUS).$^{53}$ Essentially, as the results generated by upcoming technologies become increasingly complex, concerns about automated interpretations being provided to untrained individuals with no assistance or counselling will inevitably grow, in both DTC-GT and TPI.

Overall, the goal of this paper is to promote awareness of the existence of TPI services and the ethical challenges surrounding them. TPI services represent a growing trend toward a bifurcation of services in consumer genomics - between those generating genetic data, and those offering to interpret it. ${ }^{52}$ The existence of TPI adds a new layer of complexity to the debate, and there is an urgent need to understand their implications in the DTC-GT landscape. And yet, these TPI services have been all but absent from our current dialogue. More research is needed to characterize these websites, and better understand their population of users. While formal regulation of TPI may be difficult, ${ }^{52}$ there is a need, at the very least, for better public and medical education about such resources.

\section{CONFLICT OF INTEREST}

The authors declare no conflict of interest.

\section{ACKNOWLEDGEMENTS}

LB was funded by a European Commission Bioethics Fellowship.

1 Howard HC, Borry P: Survey of European clinical geneticists on awareness, experiences and attitudes towards direct-to-consumer genetic testing. Genome Med 2013; 5: 45.

2 Nelson TN, Armstrong L, Richer J et al: CCMG statement on direct-to-consumer genetic testing. Clin Genet 2011; 81: 1 .

3 Caulfield T, McGuire AL: Direct-to-consumer genetic testing: perceptions, problems, and policy responses. Annu Rev Med 2012; 63: 23-33.

4 ESHG: Statement of the ESHG on direct-to-consumer genetic testing for health-related purposes. Eur J Hum Genet 2010; 18: 1271-1273.

5 Hudson K, Javitt G, Burke W, Byers P: ASHG Statement on direct-to-consumer genetic testing in the United States. Obstet Gynecol 2007; 110: 1392-1395.

6 ACMG: Direct-to-consumer genetic testing: a revised position statement of the American College of Medical Genetics and Genomics. Genet Med 2016; 18: 207-208.

7 Borry P, van Hellemondt RE, Sprumont D, Jales CFD, Rial-Sebbag E, Spranger TM: Legislation on direct-to-consumer genetic testing in seven European countries. Eur J Hum Genet 2012; 20: 715-721.

8 Brach M: The FDA and me. Nature 2013; 504: 7-8.
9 23andMe. https://www.23andme.com/ (accessed 4 Jan 2017).

10 FamilyTreeDNA. https://www. familytreedna.com (accessed 4 Jan 2017).

11 Ancestry.com. http://www.ancestry.com/ (accessed 4 Jan 2017).

12 STORMSEQ. http://www.stormseq.org (accessed 4 Jan 2017).

13 Promethease. https://www. promethease.com (accessed 4 Jan 2017).

14 Enlis Personal. https://www.enlis.com/personal edition.html (accessed 4 Jan 2017).

15 Genetic Genie. http://geneticgenie.org (accessed 4 Jan 2017).

16 Nutrahacker. https://www. nutrahacker.com (accessed 4 Jan 2017).

17 GedMatch. https://www.gedmatch.com/login1.php (accessed 4 Jan 2017).

18 Athletigen. https://www.athletigen.com (accessed 4 Jan 2017).

19 Swan M: DIYgenomics crowdsourced health research studies: personal wellness and preventive medicine through collective intelligence. AAAl Spring Symp - Tech Rep 2012; SS-12-05: 54-59.

20 OpenSNP. https://opensnp.org (accessed 4 Jan 2017).

21 DNA.land. https://dna.land/ (accessed 4 Jan 2017)

22 Codegen.eu. https://codegen.eu (accessed 4 Jan 2017).

23 Interpretome. http://esquilax.stanford.edu/ (accessed 4 Jan 2017).

24 LiveWello Genetics. https://livewello.com/genetics (accessed 4 Jan 2017).

25 Hunter DJ, Khoury MJ, Drazen JM: Letting the genome out of the bottle - will we get our wish? N Engl J Med 2008; 358: 105-107.

26 Yim S-H, Chung Y-J: Reflections on the US FDA's warning on direct-to-consumer genetic testing. Genomics Inf 2014; 12: 151-155.

27 Regalado A: How a wiki is keeping direct-to-consumer genetics alive. Technol Rev 2015; 118: 15-18.

28 Green RC, Berg JS, Grody WW et al: ACMG recommendations for reporting of incidental findings in clinical exome and genome sequencing. Genet Med 2013; 15: 565-574.

29 Estes R. Promethease - Genetic Health Information Alternative I DNAeXplained - Genetic Genealogy on WordPress.com. http://dna-explained.com/2013/12/30/promethease-genetichealth-information-alternative/.

30 Enlis Blog. http://www.enlis.com/blog/ (accessed 4 Jan2017).

31 Cariaso M, Lennon G: SNPedia: a wiki supporting personal genome annotation, interpretationand analysis. Nucleic Acids Res 2012; 40: 1308-1312.

32 Eng C, Sharp RR: Bioethical and clinical dilemmas of direct-to-consumer personal genomic testing: the problem of misattributed equivalence. Sci Trans/ Med 2010; 2 $17 \mathrm{~cm} 5$.

33 United States Government Accountability Office: Direct-to-Consumer Genetic Tests Misleading Test Results are Further Complicated by Deceptive Marketing and Other Questionable Practices 2010. http://www.gao.gov/assets/130/125079.pdf (accessed 4 Jan 2017).

34 Bunnik EM, Schermer MHN, Janssens ACJW: Personal genome testing: test characteristics to clarify the discourse on ethical, legal and societal issues. BMC Med Ethics 2011; 12: 11 .

35 Vayena E: Direct-to-consumer genomics on the scales of autonomy. J Med Ethics 2015; 41: 310-314.

36 ACMG Board of Directors: ACMG Statement Direct-to-consumer genetic testing: a revised position statement of the American College of Medical Genetics and Genomics. Genet Med 2016; 18: 207-208.

37 Gurwitz D, Bregman-Eschet Y: Personal genomics services: whose genomes? Eur J Hum Genet 2009; 17: 883-889.

38 Livewello Terms of Service. https://livewello.com/terms-of-service (accessed 4 Jan 2017).

39 Lewis NP, Treise D, Hsu SI, Allen WL, Kang H: DTC genetic testing companies fail transparency prescriptions. New Genet Soc 2011; 30: 291-307.

40 Codegen.eu Terms. https://codegen.eu/terms/ (accessed 18 June 2017).

41 Collier R: Genetic literacy poor in primary care. CMAJ 2012; 184: 467-468.

42 Lachance CR, Erby L A H, Ford BM, Allen VC, Kaphingst KA: Informational content, literacy demands, and usability of websites offering health-related genetic tests directly to consumers. Genet Med 2010; 12: 304-312.

43 Marietta C, Mcguire AL: Direct-to-consumer genetic testing: is it the practice of medicine? J Law Med Ethics 2009; 37: 369-374.

44 Singleton A, Erby LH, Foisie K V, Kaphingst KA: Informed Choice in Direct-toConsumer Genetic Testing (DTCGT) websites: a content analysis of benefits, risks, and Limitations. J Genet Couns 2012; 21: 433-439.

45 Cornel MC, Van El CG, Borry P: The challenge of implementing genetic tests with clinical utility while avoiding unsound applications. J Community Genet 2014; 5: 7-12.

46 Ries NM. AAnalysis of privacy policies and practices of direct-to-consumer genetic testing companies: private sector databanks and privacy protection norms. 2010, http://www.law.ualberta.ca/centres/hli/userfiles/file/Full\%20Version\%200PCC\% 20Report\%20March\%2028\%202010.pdf.

47 Codegen.eu Privacy. https://edge.codegen.eu/privacy/ (accessed 4 Jan 2017).

48 DIY Genomics. http://www. diygenomics.org (accessed 4 Jan 2017).

49 Shabani M, Borry P: Challenges of web-based personal genomic data sharing. Life Sci Soc Policy 2015; 11: 3.

50 Livewello. https://livewello.com (accessed 4 Jan 2017).

51 Karczewski KJ, Tirrell RP, Cordero P et al: Interpretome: a freely available, modular, and secure personal genome interpretation engine. Pac Symp Biocomput 2012; 339-350.

52 Spector-Bagdady K, Pike E: Consuming genomics: regulating direct-to-consumer genetic and genomic information. Neb Law Rev 2013; 92: 677-745.

53 http://digitalcommons.unl.edu/nlr/vol92/iss4/2 (accessed 18 June 2017).

54 van den Berg S, Shen Y, Jones SJM, Gibson WT: Genetic counseling in direct-toconsumer exome sequencing: a case report. J Genet Couns 2014;: 742-753. 
55 Enlis About. https://www.enlis.com/about.html (accessed 4 Jan 2017).

56 Enlis Personal Import. https://www.enlis.com/import/ (accessed 4 Jan 2017).

57 Enlis Terms and Conditions. https://www.enlis.com/import/termsandconditions.html (accessed 4 Jan 2017).

58 Enlis Privacy Policy. https://www.enlis.com/import/privacypolicy.html (accessed 4 Jan 2017).
59 LiveWello about. https://livewello.com/our-mission (accessed 4 Jan 2017).

60 https://livewello.com/privacy-policy. https://livewello.com/privacy-policy (accessed 4 Jan 2017).

61 Promethease Privacy Policy. http://www.snpedia.com/index.php/Promethease/privacy (accessed 4 Jan 2017) 